\title{
Using a Semiconductor-to-Metal Transition to Control Optical Transmission through Subwavelength Hole Arrays
}

\author{
E. U. Donev, ${ }^{1}$ J. Y. Suh, ${ }^{1}$ R. Lopez, ${ }^{2}$ L. C. Feldman, ${ }^{1,3}$ and R. F. Haglund Jr. ${ }^{1}$ \\ ${ }^{1}$ Department of Physics and Astronomy and Vanderbilt Institute of Nanoscale Science and Engineering, Vanderbilt University, \\ Nashville, TN 37235, USA \\ ${ }^{2}$ Department of Physics and Astronomy and Institute for Advanced Materials, Nanoscience and Technology, \\ University of North Carolina, Chapel Hill, NC 27514, USA \\ ${ }^{3}$ Department of Physics and Astronomy, Rutgers, The State University of New Jersey, Piscataway, NJ 08854, USA
}

Correspondence should be addressed to R. F. Haglund Jr., richard.haglund@vanderbilt.edu

Received 10 July 2007; Accepted 3 January 2008

Recommended by Stefan Maier

We describe a simple configuration in which the extraordinary optical transmission effect through subwavelength hole arrays in noble-metal films can be switched by the semiconductor-to-metal transition in an underlying thin film of vanadium dioxide. In these experiments, the transition is brought about by thermal heating of the bilayer film. The surprising reverse hysteretic behavior of the transmission through the subwavelength holes in the vanadium oxide suggest that this modulation is accomplished by a dielectric-matching condition rather than plasmon coupling through the bilayer film. The results of this switching, including the wavelength dependence, are qualitatively reproduced by a transfer matrix model. The prospects for effecting a similar modulation on a much faster time scale by using ultrafast laser pulses to trigger the semiconductor-to-metal transition are also discussed.

Copyright ( $\odot 2008$ E. U. Donev et al. This is an open access article distributed under the Creative Commons Attribution License, which permits unrestricted use, distribution, and reproduction in any medium, provided the original work is properly cited.

\section{Introduction}

Ever since its initial description in a much-cited letter by Ebbesen et al. [1], the phenomenon of extraordinary optical transmission (EOT) has generated intense interest regarding both the fundamental physics of the transmission mechanism and potential applications. In brief, the EOT effect refers to the observations that (i) light transmission through periodic arrays of subwavelength holes in opaque thin films can be much larger than the combined transmission for isolated holes predicted by standard diffraction theory [2], and (ii) the spectral profile of the transmission follows a sequence of dips and peaks peculiar to the metal and the detailed geometry of the hole array. This effect has been described predominantly in terms of light waves coupled to collective oscillations of the free electrons (surface plasmons), which bear the SPP acronym—surface plasmon polaritons.

Numerous applications of EOT have been proposed and, in some cases, reduced to practice. Specific applications of subwavelength holes [3] include SPP-activated lithographic masks, bright point sources, SPP couplers/decouplers, nearfield optical storage heads, molecular sensors, and so on. Recent work [4] has shown that the electroluminescence efficiency of an organic light-emitting diode (OLED) can be significantly enhanced by use of an perforated anode, which allows for light emission from both the back and the front of the device. Control of the EOT through a hole array could make it possible, for example, to modulate selectively in wavelength and time the light generated by an OLED or other light sources, or propagation in a waveguide, in effect serving as a subwavelength optical switch. However, we are aware of only two previous reports on schemes for postfabrication modulation of the EOT at visible and near-infrared wavelengths, and both involve varying the refractive index of the input side dielectric. Moreover, both would be difficult to implement in the kinds of applications described here. In one case, the authors [5] used different index-matching liquids to vary the degree of asymmetry between the dielectric layer above a perforated gold film and the quartz substrate. The other scheme [6] entailed sandwiching a layer of liquid crystal between a transparent 
indium-tin-oxide electrode and a perforated chromium film on quartz, then varying the applied electric field. Ultrafast switching of $\mathrm{THz}$ signals through metallic subwavelength hole arrays has recently been treated elsewhere [7].

Here we review another recently demonstrated method to control the amount of light transmitted through perforated double-layer thin films on transparent substrates $[8,9]$. The structures consist of an optically opaque metal film (silver or gold) on top of a vanadium dioxide $\left(\mathrm{VO}_{2}\right)$ film deposited on a glass $\left(\mathrm{SiO}_{2}\right)$ substrate, with a periodic array of subwavelength holes penetrating the double layer. The intensity of transmitted light is controlled by means of a reversible semiconductor-to-metal phase transition (SMT), thermally induced in the $\mathrm{VO}_{2}$ layer, which undergoes drastic changes in its electrical and optical properties. Much to our initial surprise, these structures exhibit larger optical transmission with the $\mathrm{VO}_{2}$ layer in the metallic state as compared to transmission in the semiconducting statequite the opposite of the well-known behavior of plain (i.e., no over layer, no holes) $\mathrm{VO}_{2}$ films of any thickness. This reverse optical switching can be understood in terms of a simple model that takes into account the losses due to leaky evanescent waves in the plane of the $\mathrm{VO}_{2}$ layer and diffuse scattering from the holes at the entrance and exit apertures. Numerical simulations based on the transfer matrix formalism for photonic crystals provide semiquantitative support for the experimental findings.

\section{The Extraordinary Transmission Effect and the Semiconductor-to-Metal Transition}

Many authors [5, 10-13] have elaborated on the initial explanation (given in [1]) that the excitation of surfaceplasmon polaritons (SPPs) at either or both metal-dielectric interfaces mediates the EOT effect. A small group of researchers had previously dismissed the role of SPPs and put forth a model of nonresonant generation and interference of composite diffracted evanescent waves (CDEWs) [14]; however, a more recent exchange of articles, [15-19], comments $[20,21]$, and responses to comments [22, 23] between proponents of the SPP and CDEW models has led to a tacit consensus that surface plasmons must be involved in the extraordinary transmission at visible and near-infrared wavelengths. Indeed, most workers in this field now regard the excitation of propagating SPP modes as the dominant mechanism for mediating the EOT effect in metal films perforated by periodic arrays of subwavelength apertures. Recent studies [24, 25] have also identified another, albeit weaker, contribution to the observed transmission, namely, the localized surface-plasmon resonances [26] (LSPRs) that originate on the metallic ridge of each aperture. Revealing the role of the LSPRs may in fact has provided the concrete evidence to explain why hole arrays in good metals such as gold and silver invariably exhibit the largest EOT effects [24]. Other researchers [27-29] have interpreted the EOT effect in a purely phenomenological fashion-in terms of Fanotype spectral profiles, which result from the interference of two distinct contributions: a resonant channel (e.g., SPPs and/or other surface modes) and nonresonant scatteringand accounted for the observed spectral shapes without explicitly specifying the nature of the resonant channel.

The EOT spectra exhibit very sharp minima in the transmission profiles. These minima have routinely been attributed to the nonresonant Wood's anomaly for diffraction gratings [30], which amounts to the disappearance of a diffracted order as it becomes tangent to the plane of the grating. The spectral positions of the minima are known as Rayleigh wavelengths. At normal incidence, the first-order Rayleigh wavelength coincides with the grating spacing [31].

Concerning $\mathrm{VO}_{2}$ and its semiconductor-to-metal transition (SMT), it is a first-order phase transition, and occurs at a critical temperature $T_{c} \approx 67^{\circ} \mathrm{C}$, from a high-temperature metallic phase to a low-temperature semiconducting phase. Above $T_{c}, \mathrm{VO}_{2}$ has a tetragonal rutile structure and exhibits metallic character; of particular interest here is the relatively high optical opacity, especially in the infrared (IR) wavelength range. Below $T_{c}$, the dimerization and tilting of the $\mathrm{V}-\mathrm{V}$ pair result in a monoclinic unit cell and the opening of a narrow band-gap [32]; in this phase, the film is markedly more transparent than it is in the metallic phase (Figure 3(d)). The precise mechanism of the phase transition has long been a topic of controversy; whether the SMT is driven by electron correlations or by the band-gap opening (for a review, see [33]). In fact, a recent theoretical study [34] concludes that an interplay of charge and lattice degrees of freedom initiates and drives the SMT. Both components of the transition (lattice and electronic) take place in less than $100 \mathrm{fs}$, which makes $\mathrm{VO}_{2}$ a candidate for applications in fast optical switching [35]. The presence of a thermal hysteresis (Figure 3(d)), which is attributed to variations in the phaseequilibrium temperatures for film grains of different sizes [36], allows for potential applications in memory devices and optical data storage.

\section{Experimental Method and Results}

Two types of structures investigated in $[8,9]$ were: (i) hole array in Ag-on- $\mathrm{VO}_{2}$ double layer on glass (Figure 1(b)); (ii) hole array in Au-on- $\mathrm{VO}_{2}$ on glass (Figure 1(b)); (iii) hole array in $\mathrm{VO}_{2}$ film on glass (Figure $1(\mathrm{c})$ ); (iv) plain $\mathrm{VO}_{2}$ film on glass. All the $\mathrm{VO}_{2}$ layers were prepared simultaneously on fused-silica substrates in a pulsed-laser deposition (PLD) system (KrF excimer laser: $\lambda=248 \mathrm{~nm}$, fluence $\approx 4 \mathrm{~J} / \mathrm{cm}^{2}$, repetition rate $=25 \mathrm{~Hz}$ ) by ablating a vanadium target in a $12-\mathrm{mTorr}_{2} \mathrm{O}_{2}$ atmosphere at $550^{\circ} \mathrm{C}$. The resulting film thickness was $200 \mathrm{~nm}$, as determined by Rutherford backscattering spectrometry (RBS). The Ag and Au overlayers were deposited in a bell-jar thermal evaporator. RBSmeasured thicknesses were $160 \mathrm{~nm}$ for $\mathrm{Ag}$ and $230 \mathrm{~nm}$ for $\mathrm{Au}$. The hole arrays, consisting of $60 \times 60$ nominally cylindrical apertures of 250-nm diameter and 750-nm pitch, were milled down to the substrates with a focused ion beam (FIB: liquid $\mathrm{Ga}^{+}$ion source at $30 \mathrm{keV}, 90-\mathrm{pA}$ beam current). Figure 1(a) shows an FIB micrograph of part of such an array (structure type (iii)), while Figure 1(d) shows the same array (different portion) imaged in illumination mode with a scanning nearfield optical microscope (SNOM: $\lambda=532 \mathrm{~nm}$, aperture 


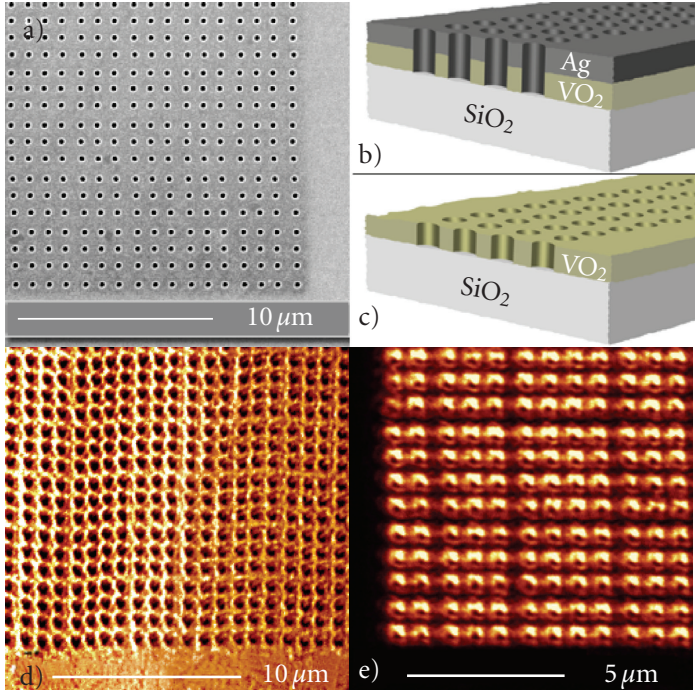

FIgURE 1: (Color online) (a) FIB micrograph of a hole-array corner. Schematics of the studied hole arrays in: (b) $\mathrm{Ag} / \mathrm{Au}-\mathrm{VO}_{2}$ double layers, (c) single $\mathrm{VO}_{2}$ layer. SNOM transmission images $(\lambda=$ $532 \mathrm{~nm}$ ) of hole arrays in: (d) single $\mathrm{VO}_{2}$ layer (semiconducting phase), (e) $\mathrm{Au}-\mathrm{VO}_{2}$ double layer. All perforated structures are on glass substrates.

diameter $\approx 100 \mathrm{~nm})$. It is relevant to note here that the airfilled holes in this SNOM image appear darker, that is, transmit less than the surrounding $\mathrm{VO}_{2}$ film, whereas the contrast is reversed in the case of holes in $\mathrm{Ag}-\mathrm{VO}_{2}$ (Figure 1(e)) or $\mathrm{Au}-\mathrm{VO}_{2}$ double layers (not shown). We shall return to this observation shortly. Spectral measurements, at normal and oblique incidence, were performed with collimated white light through an optical fiber and a micro-objective. The incident-beam spot was slightly smaller than the size of the array, and the beam divergence was determined to be $\approx 1^{\circ}$. The transmission spectrum through each sample was collected in the zero-diffraction-order (i.e., detected beam collinear with incident beam) by another micro-objective, stopped down to reduce outgoing-beam divergence to $\approx 1^{\circ}$, and coupled to an optical fiber. The fiber was then fed into a monochromator equipped with a cooled-CCD detector. The substrates were attached to a resistor-heated sample holder, mounted on a translation-rotation stage, with a precision thermocouple placed in contact with the top surface of the sample. The position of the hole array with respect to the incident beam was monitored in reflection via a CCD camera connected to a video display.

Figure 2 presents the main experimental results of the work in [8]: (i) controlled switching of the zero-order extraordinary optical transmission (EOT) through periodic subwavelength structures, and (ii) highly unusual transmission behavior of the $\mathrm{VO}_{2}$ layer in these structures. Figure 2(a) shows the transmission spectra for a hole array in a silver$\mathrm{VO}_{2}$ double layer on glass, at two different temperatures, which correspond to the two phases of the $\mathrm{VO}_{2}$ layer. It is immediately obvious that, for wavelengths greater than about $600 \mathrm{~nm}$, the high-temperature (i.e., $\mathrm{VO}_{2}$ in its metallic phase) transmission dominates. The transmission contrast in the visible range is rather small, at some points even slightly in favor of the low-temperature transmission (i.e., $\mathrm{VO}_{2}$ in its semiconducting phase), since the optical properties of the semiconducting and metallic phases of $\mathrm{VO}_{2}$ differ considerably only in the IR range, as evidenced by the spectral dependence of the complex permittivity (Figure 3(c) and inset; data from [37]) and the transmission (Figures 3(a) and $3(\mathrm{~b})$, solid lines). (Figure 2(a), inset) shows the zeroorder EOT intensity as a function of temperature at $800 \mathrm{~nm}$. Comparing the shape of this hysteresis curve to that of a plain $\mathrm{VO}_{2}$ film (Figure 3(d)), it becomes clear why we call our observation reversed optical switching of $\mathrm{VO}_{2}$. In all fairness, some credit should go to the air-filled holes, since it is really the permittivity contrast between the content of the holes (air) and the surrounding material $\left(\mathrm{VO}_{2}\right)$ that contributes to the effect, as we explain later on.

The controlled switching of the zero-order EOT in the near-IR range stands out even more prominently in the case of a perforated gold- $\mathrm{VO}_{2}$ double layer on glass (Figure 2(b)) - for example, the high-temperature transmission at $800 \mathrm{~nm}$ exceeds the low-temperature transmission by more than a decade. The SNOM image (Figure 1(e)) clearly shows that virtually no light can pass through the intact film and that all the detected light must have traveled through the holes. Similar images were obtained for the hole arrays in $\mathrm{Ag}-\mathrm{VO}_{2}-\mathrm{SiO}_{2}$. The overall magnitude of the zeroorder EOT through the gold $-\mathrm{VO}_{2}-\mathrm{SiO}_{2}$ structure is smaller than the magnitude in the corresponding phase of the silver- $\mathrm{VO}_{2}-\mathrm{SiO}_{2}$ structure, most likely because of the larger metal-layer thickness [38] and smaller effective hole diameter [39] for the gold-structure with respect to the silver structure. The relative sharpness of the maximum transmission peak for the gold $-\mathrm{VO}_{2}$ structure might also be related to hole size: smaller holes, being less efficient scatterers of surface modes into far-field light, diminish the radiative damping and hence increase the lifetime of these modes, which in turn reduces the width of the peak [40]. In any case, the key observation for both types of structures remains that EOT in the metallic phase of $\mathrm{VO}_{2}$ is further enhanced over EOT in the semiconducting phase.

As noted above, at normal incidence, the first-order Rayleigh wavelength coincides with the grating spacing, or in this case, with the hole spacing in the array [31]. The sharp normal-incidence minima for our samples occur close to $750 \mathrm{~nm}$ (Figures 2(a) and 2(b)), which corresponds to the nominal lattice spacing for our hole arrays. As the incidence angle is increased, the Rayleigh wavelengths for grazing orders should become longer on one side of the sample normal and become shorter on the other side [31]. Consequently, we expect the sharp minimum at $\lambda(\mathrm{R})$ to split into two separate minima: one occurring at $\lambda(\mathrm{R}-)<\lambda(\mathrm{R})$, and the other at $\lambda(\mathrm{R}+)>\lambda(\mathrm{R})$. Indeed, our experimental observations confirm this prediction. Figure 2(c) shows the doubling of the normal-incidence Wood's anomaly as the sample plane is rotated with respect to the incident-detectedbeam axis. The polarization of the incident light was such that the electric field would oscillate parallel to the rows (or columns) of the $\mathrm{Ag}-\mathrm{VO}_{2}-\mathrm{SiO}_{2}$ hole array. Both the high- and 


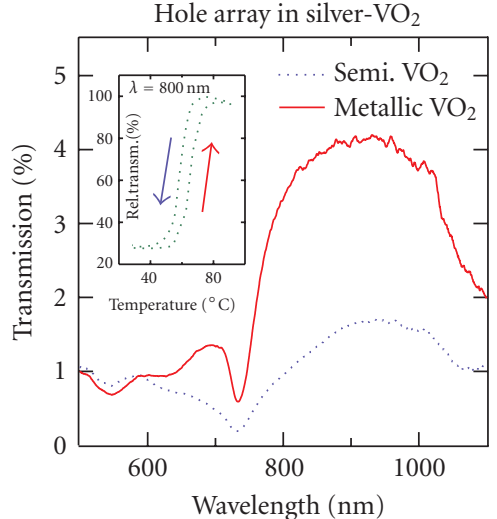

(a)

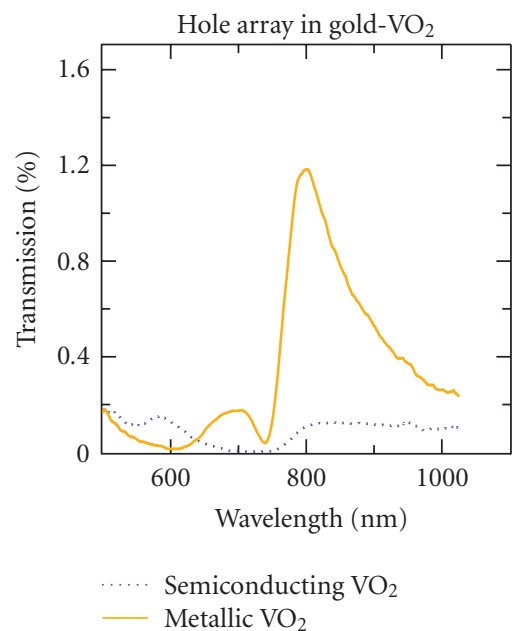

(b)

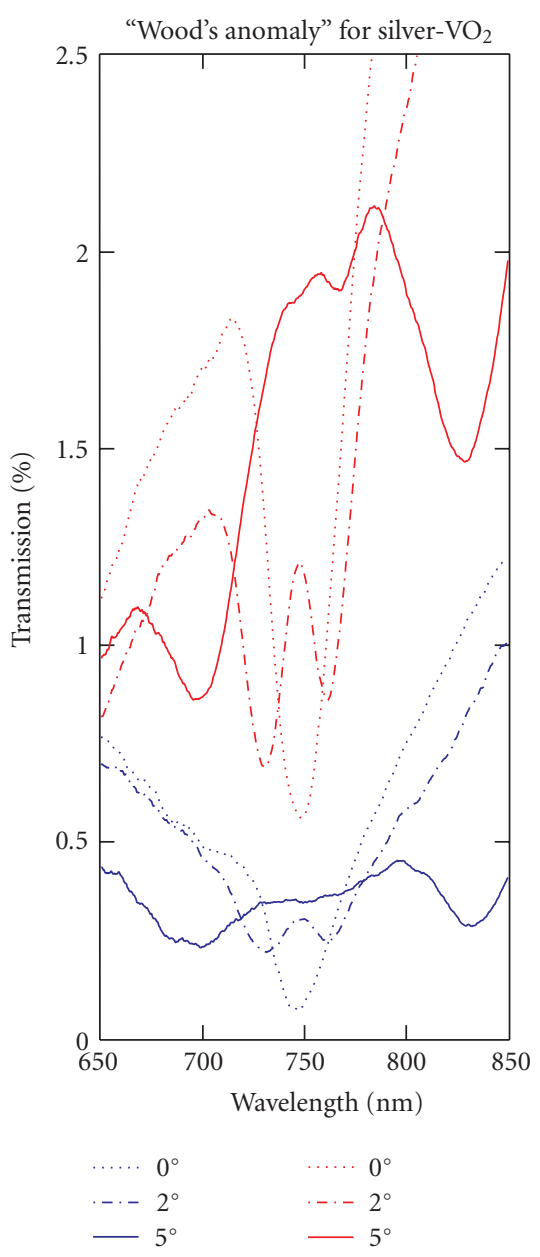

(c)

FIGURE 2: (Color online) experimental zero-order transmission spectra, in each $\mathrm{VO}_{2}$ phase, for perforated double layers in: (a) $\mathrm{Ag}$ - $\mathrm{VO}_{2}$, with (inset) its reverse hysteresis at $\lambda=800 \mathrm{~nm}$ (normalized to the highest transmission in the metallic phase of the $\mathrm{VO}_{2}$ layer; upward/downward arrow denotes the heating/cooling part of the thermal cycle; cf, regular hysteresis in Figure 3(d)), (b) Au- $\mathrm{VO}_{2}$, (c) demonstration of the spectral splitting of the Wood's anomaly minima with deviation from the normal $\left(0^{\circ}\right)$ angle of incidence (lower/upper set of three curves corresponds to the semiconducting/metallic phase of the $\mathrm{VO}_{2}$ layer in the $\mathrm{Ag}-\mathrm{VO}_{2}$ hole array).

low-temperature phases exhibit two sharp dips in each of their transmission curves for nonzero angles of incidence. For instance, the zero-order transmission at incidence angle of $2^{\circ}$ has one dip at $730 \mathrm{~nm}$ and another one at $760 \mathrm{~nm}$, as opposed to a single dip at $750 \mathrm{~nm}$ for the zero-order transmission at $0^{\circ}$. In addition, we find once again that the EOT in the high-temperature state - for all pairs of curvesis further enhanced over the EOT in the low-temperature state.

Returning to the reverse-switching effect, the following explanation has been proposed. Since the metal film (Ag or Au) is optically thick, none of the incident light can traverse the nonperforated double-layer structure (Figure 1 (e), black regions). Therefore, transmission can only occur through the holes in the metal layer, in the form of evanescent waves generated by diffraction of the incident light at the metal-air interface and mediated by surfaceplasmon polaritons.
The waves that emanate from the holes in the metal overlayer undergo additional scattering and must then travel through the perforated $\mathrm{VO}_{2}$ layer, where they become leaky evanescent waves, that is, waves that lose intensity as they propagate [41]. The $\mathrm{VO}_{2}$ acts as a lossy medium: the waves penetrate the side walls of the air-filled holes and leak into the plane of the $\mathrm{VO}_{2}$ layer, where partial absorption occurs. As a result, this leakage channels a portion of the light away from the zero-order transmission path and thus renders it undetected in the far field. The amount of light that penetrates into the $\mathrm{VO}_{2}$ material between the holes depends on the optical constants of metallic or semiconducting $\mathrm{VO}_{2}$ and, in particular, on the permittivity contrast between the hole content (air) and its surroundings $\left(\mathrm{VO}_{2}\right)$. Lower permittivity contrast reduces both the leakage into the $\mathrm{VO}_{2}$ layer and the nonforward (diffuse) scattering from the holes at the air and glass interfaces [42]; conversely, higher permittivity contrast increases those losses. 


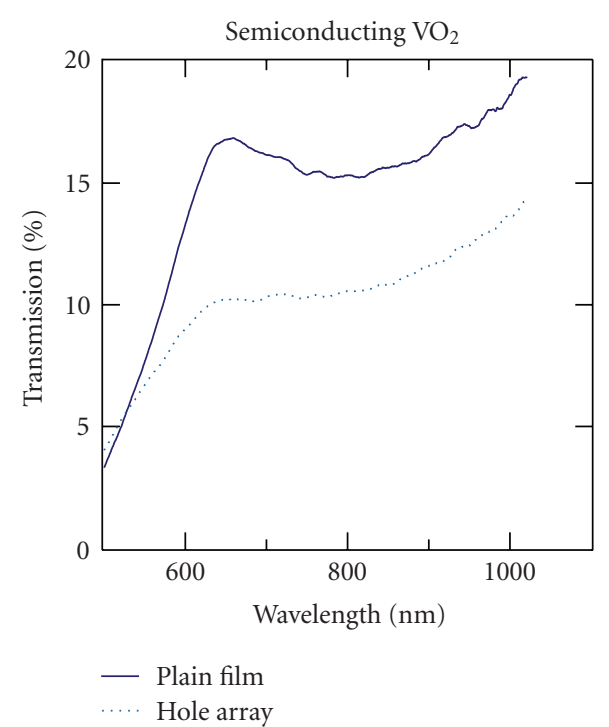

(a)

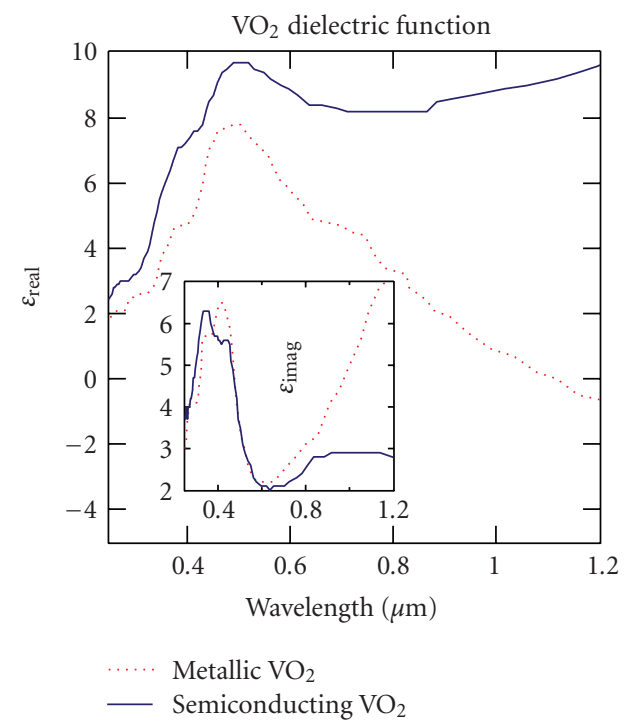

(c)

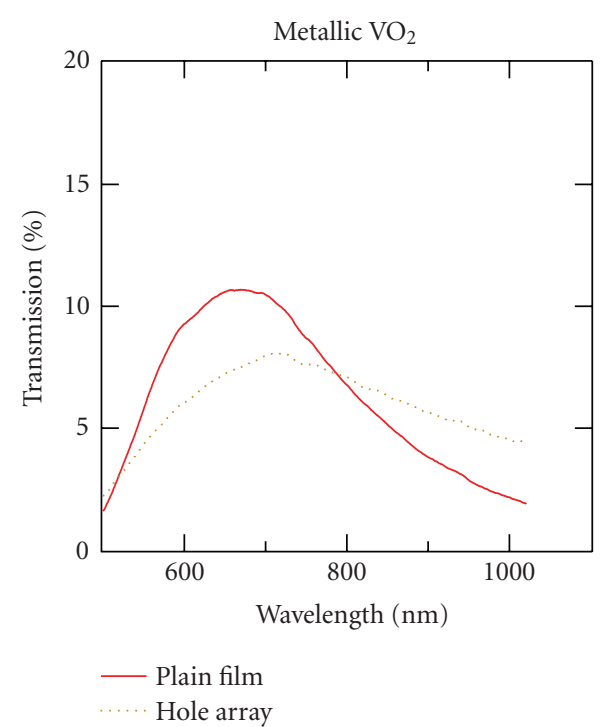

(b)

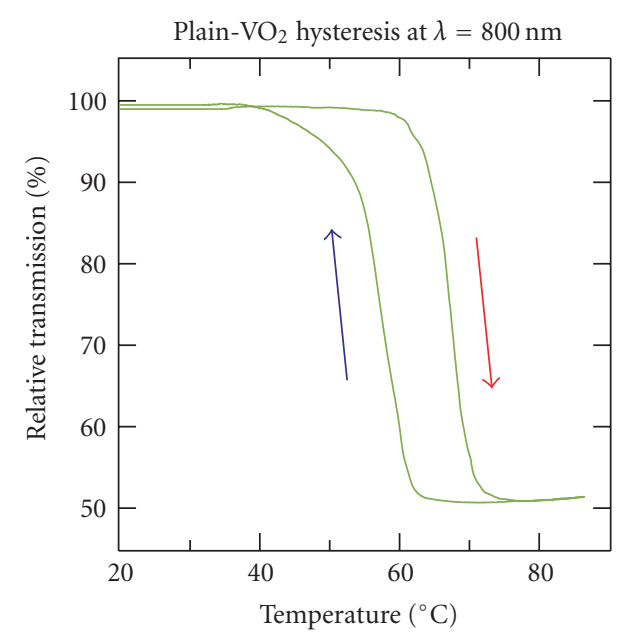

(d)

FIgURE 3: (Color online) experimental zero-order transmission spectra for single plain (i.e., no holes) and perforated layers in: (a) semiconducting $\mathrm{VO}_{2}$, (b) metallic $\mathrm{VO}_{2}$. Wavelength dispersion of the (c) real and (inset) imaginary parts of the $\mathrm{VO}_{2}$ dielectric function, in each phase (data extracted from [24]), (d) Regular hysteresis at $\lambda=800 \mathrm{~nm}$ for the plain- $\mathrm{VO}_{2}$ case (normalized to the highest transmission in the semiconducting phase of the $\mathrm{VO}_{2}$ layer; downward/upward arrow denotes the heating/cooling part of the thermal cycle; cf, reverse hysteresis in (Figure 2(a) inset).

In the visible range (e.g., up to $500 \mathrm{~nm}$ ), the complex permittivities of the two phases of $\mathrm{VO}_{2}$ (Figure $3(\mathrm{c})$ and inset) exhibit similar trends and values, so the transmission contrast between the two phases remains relatively low in this wavelength range (Figures 2(a), 2(b) and 3(a), $3(\mathrm{~b}))$. In the near-IR range, however, the permittivity of metallic $\mathrm{VO}_{2}$ differs significantly from its semiconducting counterpart; for example, at $850 \mathrm{~nm} \varepsilon\left(\mathrm{VO}_{2}\right.$-met $)=2.67+$ $2.98 i$, while $\varepsilon\left(\mathrm{VO}_{2}\right.$-semi $)=8.17+2.65 i$. Therefore, because the permittivity contrast between the interior (air) and the exterior $\left(\mathrm{VO}_{2}\right)$ of the holes is lower in the case of metallic
$\mathrm{VO}_{2}$, the zero-order high-temperature transmission receives an enhancement over the zero-order low-temperature transmission.

In order to bring out the significance of the $\mathrm{VO}_{2}$ layer for the metallic-phase enhancement of the EOT in $\mathrm{Ag}-\mathrm{VO}_{2}-\mathrm{SiO}_{2}$ and $\mathrm{Au}-\mathrm{VO}_{2}-\mathrm{SiO}_{2}$ structures, the optical properties of a hole array in $\mathrm{VO}_{2}$ on glass (Figure 1(c)), that is, without a goodmetal overlayer, were also examined [9]. It must be noted that transmission observed through this type of structure is not extraordinary, in the sense used thus far, owing to the insufficient opacity of the $\mathrm{VO}_{2}$ layer. Figure 3(a) compares 
the zero-order transmission, in the semiconducting phase, through the hole array (dotted line) and through a nonperforated area nearby (solid line). The hole array transmits less than the intact film throughout the wavelength range because the array serves as a diffraction grating that diverts some of the incident power into nonzero diffracted orders, which propagate out of the detector path and consequently do not contribute to the zero-order transmission. Similarly to the loss mechanisms discussed above, a portion of the diffracted field becomes trapped as leaky waves in the plane of the $\mathrm{VO}_{2}$ layer, while some of the light that persists in the zero order inside the holes undergoes additional diffuse scattering at the glass interface. Also, since direct transmission through the partially transparent $\mathrm{VO}_{2}$ film overwhelms the transmission emerging from the holes, the holes appear darker than their surroundings in the SNOM image of low-temperature transmission through a $\mathrm{VO}_{2}$ array (Figure 1(d)), as mentioned earlier.

Figure 3(b) presents corresponding transmission curves for the metallic phase of the $\mathrm{VO}_{2}$ layer. Here, something intriguing happens in the near-IR range: in spite of diffraction effects, the zero-order transmission through the hole array (dotted line) exceeds the direct transmission through the flat film (solid line). This observation also pertains to the above argument that the air-filled holes, when surrounded by metallic- $\mathrm{VO}_{2}$, tend to channel the light along the forward direction by reducing the losses due to leaky waves and nonforward scattering, all to the benefit of the detected zero-order transmission. Part of the light traversing the perforated film passes through air-filled holes instead of passing through $\mathrm{VO}_{2}$ material, and so it avoids the absorption and reflection that befall all the light traversing the flat $\mathrm{VO}_{2}$ film. Ultimately, in the metallic- $\mathrm{VO}_{2}$ case, the zero-order transmission through the hole array prevails over the direct transmission through the nonperforated film.

A major point in this qualitative explanation is that the holy films with nanoscale dimensions, with or without the metal, result in a very large effective interfacial area for the transmitted photons. This effect is a direct property of the ability to construct these nanoscale structures.

\section{Numerical Simulations}

Numerical simulations of the zero-order transmission spectra through all four types of experimental structures were undertaken to see if they would reproduce the general features observed in the experiments of $[8,9]$ (Figure 4). The computational scheme stems from a numerical method for modeling the properties of one- and two-dimensional patterned photonic crystal slabs. All of our simulations use the optical constants of $\mathrm{VO}_{2}$ as extracted from [37] and plotted in Figure 3(c); the optical constants of Ag and $\mathrm{Au}$ were taken from [43]. The well-known transfer matrix formalism was employed [44]: (i) the piecewise complex permittivity within each layer is represented by a Fourier decomposition in terms of reciprocal square lattice vectors; (ii) the solution of Maxwell's equations in each layer is decomposed into sets of eigenvectors propagating along the normal to the slab, in both directions; (iii) transfer matrices connect the amplitudes of partial waves at different planar slices of the slab inside the same layer; (iv) interface matrices connect the amplitudes across successive layers of the slab by imposing continuity conditions on the tangential (i.e., inplane) components of the electric and magnetic fields at the interface; (v) material matrices convert the partial-wave amplitudes at a given point into inplane components of electric and magnetic fields at this point.

In essence, this method constructs a total transfer matrix that connects the field amplitudes at different dielectric planes. The first task in this scheme is to perform Fourier transformations of the two-dimensional periodic dielectric function, $\vec{\varepsilon}(\vec{r})$, for the reciprocal lattice vectors, $\vec{G}$, assuming the function is only dependent on $x$ and $y$ as

$$
\begin{aligned}
\mathcal{E}_{\vec{G} \vec{G}^{\prime}} & =\frac{1}{S} \int \mathcal{E}(\vec{r}) \exp \left[i\left(\vec{G}^{\prime}-\vec{G}\right) \vec{r}\right] d x d y, \\
\vec{G} & =\left(\frac{2 \pi g_{x}}{d_{x}}, \frac{2 \pi g_{y}}{d_{y}}\right), \quad g_{x}=g_{y}=0, \pm 1, \pm 2, \ldots, \pm g_{\max }
\end{aligned}
$$

where $d_{x}$ and $d_{y}$ are the periods along $x$ - and $y$-axis, and each layer is assumed to have translational invariance along $z$-axis. The numerical calculation implements this integral over a finite subset of the reciprocal vectors, with $N_{g}=\left(2 g_{x, \max }+\right.$ 1) $\left(2 g_{y, \max }+1\right)$ elements. This Fourier decomposition can be evaluated so that the order of convergence is smaller than $10^{-3}$. Next, the solution of Maxwell's equations is written as a Fourier decomposition into a sum of plane waves:

$$
\vec{E}(x, y, z, t)=\overrightarrow{E_{G}} \exp \left(i k_{x, \vec{G}} x+i k_{y, \vec{G}} y\right) \exp (i K z-i \omega t) .
$$

Here, $k_{x, \vec{G}}$ is defined as $k_{x, \vec{G}}=k_{x}+G_{x}$, where $k_{x}$ (= $\omega$ / $c \sin \theta \cos \varphi)$ is the $x$-component of the incoming wavevector, $\vec{k}$. Maxwell's equations become the eigenvalue-eigenvector problem solving $2 N_{g}$ linear equations for inplane electric components $E_{x, G}$ and $E_{y, G}$. It can be written in a compact form as

$$
(\mathbf{N})^{-1} \mathbf{M} \overrightarrow{E_{/ /}}=K^{2} \overrightarrow{E_{/ /}}
$$

The $\mathbf{M}$ and $\mathbf{N}$ are $2 N_{g} \times 2 N_{g}$ matrices that explicitly consist of the components of the wavevectors and reciprocal lattice vectors. Then, the eigenvalue $K$ that is an element of a diagonal matrix and the corresponding eigenvector $\overrightarrow{E_{/ /}}$can be computed.

At this point, two kinds of transfer matrices need to be formulated. The layer matrix (the first kind) allows us to compute the amplitude vector from $z$ to $z+L$ as follows:

$$
\vec{A}(z+L)=\overrightarrow{\mathbf{T}_{L}} \vec{A}(z), \quad \overrightarrow{\mathbf{T}_{L}}=\left(\begin{array}{cc}
\exp [i K L] & 0 \\
0 & \exp [-i K L]
\end{array}\right)
$$

The interface matrix represents the amplitude transfer across the interface between two adjacent layers of the slab. From the boundary condition of tangential components of electric 


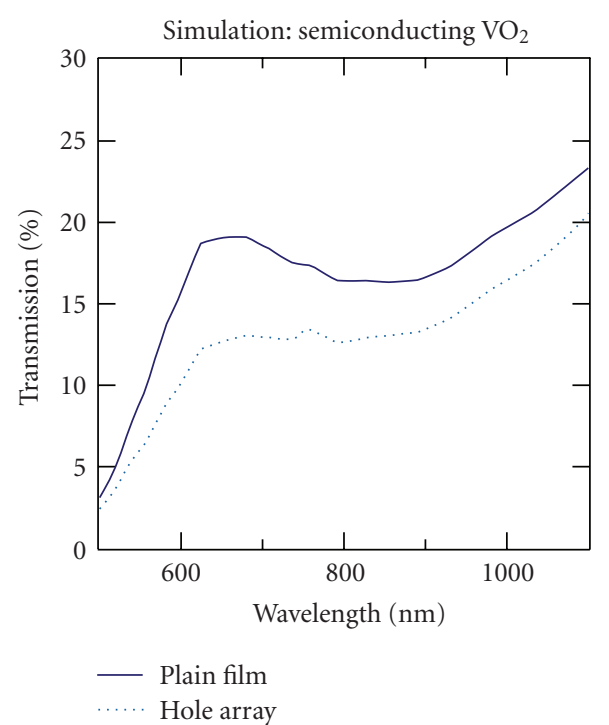

(a)

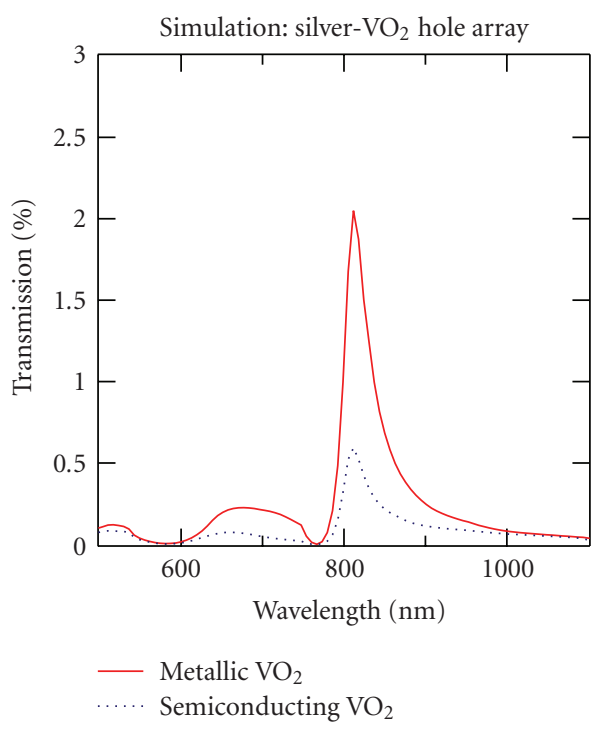

(c)

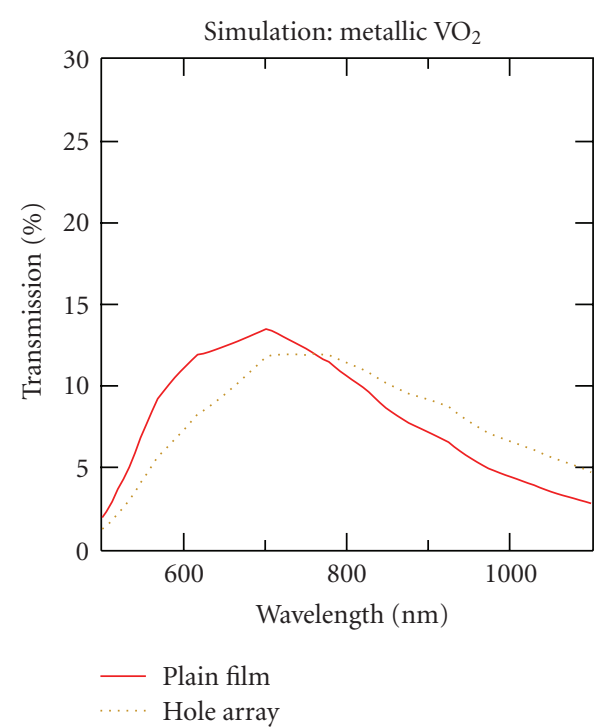

(b)

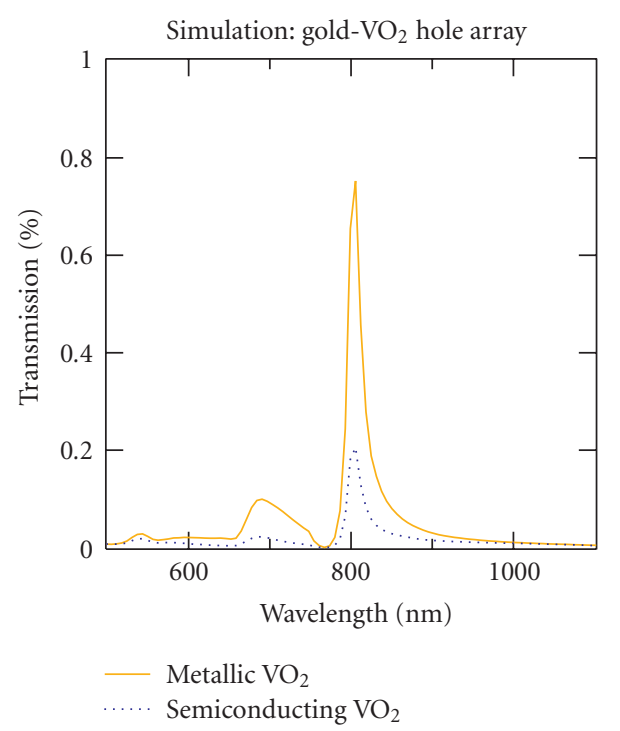

(d)

FIGURE 4: (Color online) numerical simulations of zero-order transmission, using the transfer matrix formalism (see text) and the $\mathrm{VO}_{2}$ dielectric function in Figure 3(c). Single plain (i.e., no holes) and perforated $\mathrm{VO}_{2}$ layers on glass: (a) semiconducting phase, (b) metallic phase. Perforated double layers on glass, in each $\mathrm{VO}_{2}$ phase: (c) $\mathrm{Ag}-\mathrm{VO}_{2}$, (d) $\mathrm{Au}-\mathrm{VO}_{2}$.

and magnetic fields at the interface, the interface matrix is calculated as

$$
\overrightarrow{\mathbf{T}}_{b, a}=\overrightarrow{\mathbf{F}}_{b}^{-1} \overrightarrow{\mathbf{F}}_{a}, \quad \overrightarrow{\mathbf{F}}=\left(\begin{array}{cc}
\overrightarrow{\mathbf{E}}_{/ /} & \overrightarrow{\mathbf{E}}_{/ /} \\
\frac{c}{\omega} \mathbf{C} \overrightarrow{\mathbf{E}}_{/ / \mathbf{K}}-\frac{c}{\omega} \mathbf{C} \overrightarrow{\mathbf{E}}_{/ / \mathbf{K}}
\end{array}\right),
$$

where $\mathbf{C}$ is a $2 N_{g} \times 2 N_{g}$ matrix composed of the matrix $\mathbf{N}$. Now, we can set up a total transfer matrix that describes the change of input amplitude through an entire layered-system as follows:

$$
\vec{A}_{\text {out }}=\overrightarrow{\mathbf{T}}_{\text {tot }} \vec{A}_{\text {in }},
$$

with

$$
\overrightarrow{\mathbf{T}}_{\text {tot }}=\mathbf{T}_{b, a_{N}} \mathbf{T}_{L_{N}} \mathbf{T}_{a_{N} a_{N-1}} \cdots \mathbf{T}_{L_{1}} \mathbf{T}_{a_{1}, a_{0}},
$$

where $N$ runs through the number of different layers. (E.g., in the bilayer case, the total transfer matrix comprises two layer matrices and three interface matrices.) From knowledge of the total transfer matrix and given a frequency of incoming light, the reflection, absorption, and transmission can be computed from the output electromagnetic field components. The computation is then repeated throughout the whole spectral range. 
Despite several simplifications to the model, such as square instead of circular apertures, the simulation results show good semiquantitative agreement with the corresponding experimental findings. For example, Figures 4(a) and 4(b) supports the above discussion regarding the zero-order transmission through a $\mathrm{VO}_{2}$ hole array as compared to the transmission through a flat $\mathrm{VO}_{2}$ film. In particular, the simulated curves for the metallic phase (Figure 4(b)) show the characteristic cross-over in the near-IR region, where the perforated metallic- $\mathrm{VO}_{2}$ film transmits more per unit area thanthe nonperforated (plain) film. The simulations also corroborate the key experimental observation of this work, namely, that the EOT through subwavelength hole arrays in $\mathrm{Ag}-\mathrm{VO}_{2}-\mathrm{SiO}_{2}$ (Figure 4(c)) and $\mathrm{Ag}-\mathrm{VO}_{2}-\mathrm{SiO}_{2}$ (Figure 4(d)) structures exhibits a pronounced enhancement in the nearIR range for the metallic over the semiconducting phase of the $\mathrm{VO}_{2}$ layer. Even though the calculated spectra differ somewhat in shapes and absolute magnitudes from the corresponding experimental curves, the simulations unequivocally demonstrate the effect of reverse optical switching.

\section{Prospects for All-Optical Modulation of the EOT}

Since the electronic response of the plasmonic material in these structures is fast on the femtosecond time scale, it is logical to ask whether the EOT can likewise be modulated on an ultrafast time scale. Experiments by Cavalleri et al. on $\mathrm{VO}_{2}$ films show that hole doping initiated by a femtosecond laser pulse can initiate the SMT on a time scale of a few hundred femtoseconds [45]. The details of the ultrafast response, however, are dependent on the thickness of the film. More recent X-ray studies seem to indicate that for $800 \mathrm{~nm}$ incident laser light, the turn-on response of the film is extremely fast for film thicknesses of $70 \mathrm{~nm}$ or less, but diffusive after that [46]. This ultrafast response depth is, of course, a function of the excitation wavelength, and it is possible that a laser wavelength with greater penetration depth in $\mathrm{VO}_{2}$ could achieve ultrafast SMT initiation for thicker films. The response time also appears to be a function of fluence, with important dynamical features showing a differentiated response below the 100-fs time scale [47].

The more challenging question for ultrafast modulation is whether or not the SMT can be turned off on a fast time scale. Evidence so far indicates that the return from metallic to the semiconducting phase occurs on nanosecond or subnanosecond time scales, governed essentially by thermal diffusivity $[48,49]$. While the metallic state relaxes on a subpicosecond time scale for near-threshold densities of photoinitiated electron-hole pairs, the relaxation times increase significantly with increasing electron-hole plasma density, even for rather thin $\mathrm{VO}_{2}$ films on diamond substrates [50]. In the case of the bilayer plasmonic structures embodied in our hole arrays, however, it is possible that the thermal conductivity of the metal would significantly assist the cooling transition back to the semiconducting state. Hence the question of possible ultrafast turn-on and turn-off of plasmonic effects remains an open question.

\section{Conclusions}

In generic terms, the future of optoelectronic devices relies on the ability to manipulate light on a subwavelength scale, where diffraction-limited optical elements lose their utility. Here we have reviewed a novel way to control the extraordinary optical transmission through subwavelength hole arrays in structures composed of a $\mathrm{VO}_{2}$ thin film sandwiched between an opaque metal layer and a transparent substrate. While the control mechanism here is the thermally induced semiconductor-to-metal transition of $\mathrm{VO}_{2}$, but the transition can take place on a sub-picosecond time scale if triggered by a laser pulse, which opens the possibility for ultrafast switching devices. Furthermore, the present work has uncovered a counterintuitive trend in the nearIR transmission properties of the perforated $\mathrm{VO}_{2}$ layer when compared to a continuous $\mathrm{VO}_{2}$ film of the same thickness, namely, reverse optical switching, whereby the zero-order transmitted intensity from perforated $\mathrm{Ag}-\mathrm{VO}_{2}$ or $\mathrm{Au}-\mathrm{VO}_{2}$ double layers on glass is in fact considerably higher during the metallic phase of the $\mathrm{VO}_{2}$ than it is during the semiconducting phase. A simple heuristic model is sufficient to account qualitatively for this effect, based on the idea that the losses in the zero-order transmission are caused by evanescent waves that leak into the $\mathrm{VO}_{2}$ layer, in addition to diffuse scattering at the entrance and exit apertures. The magnitude of these increases with higher permittivity contrast between the interior and the exterior of the holes. The role of metallic $\mathrm{VO}_{2}$ in further enhancing the EOT from perforated silver or gold films has been emphasized by comparing measurements on perforated and nonperforated areas of the same $\mathrm{VO}_{2}$ film, which showed that the zeroorder high-temperature transmission through a $\mathrm{VO}_{2}$ hole array can exceed the direct transmission through the flat film.

\section{Acknowledgment}

Research at Vanderbilt was supported by the National Science Foundation through a Nanoscience Interdisciplinary Research Team Grant no. (DMR-0210785) and by the United States Department of Energy Office of Science (DE-FG0201ER45916).

\section{References}

[1] T. W. Ebbesen, H. J. Lezec, H. F. Ghaemi, T. Thio, and P. A. Wolff, "Extraordinary optical transmission through subwavelength hole arrays," Nature, vol. 391, no. 6668, pp. 667$669,1998$.

[2] C. J. Bouwkamp, "Theoretical and numerical treatment of diffraction through a circular aperture," IEEE Transactions on Antennas and Propagation, vol. 18, no. 2, pp. 152-176, 1970.

[3] J. Dintinger, A. Degiron, and T. W. Ebbesen, "Enhanced light transmission through subwavelength holes," MRS Bulletin, vol. 30, no. 5, pp. 381-384, 2005.

[4] C. Liu, V. Kamaev, and Z. V. Vardeny, "Efficiency enhancement of an organic light-emitting diode with a cathode forming two-dimensional periodic hole array," Applied Physics Letters, vol. 86, no. 14, 3 pages, 2005, Article ID 143501. 
[5] A. Krishnan, T. Thio, T. J. Kim, et al., "Evanescently coupled resonance in surface plasmon enhanced transmission," Optics Communications, vol. 200, no. 1-6, pp. 1-7, 2001.

[6] T. J. Kim, T. Thio, T. W. Ebbesen, D. E. Grupp, and H. J. Lezec, "Control of optical transmission through metals perforated with subwavelength hole arrays," Optics Letters, vol. 24, no. 4, pp. 256-258, 1999.

[7] E. Hendry, M. J. Lockyear, J. Gómez Rivas, L. Kuipers, and M. Bonn, "Ultrafast optical switching of the THz transmission through metallic subwavelength hole arrays," Physical Review $B$, vol. 75, no. 23, 5 pages, 2007, Article ID 235305.

[8] J. Y. Suh, E. U. Donev, R. Lopez, L. C. Feldman, and R. F. Haglund Jr., "Modulated optical transmission of subwavelength hole arrays in metal- $\mathrm{VO}_{2}$ films," Applied Physics Letters, vol. 88, no. 13, 3 pages, 2006, Article ID 133115.

[9] E. U. Donev, J. Y. Suh, F. Villegas, R. Lopez, R. F. Haglund Jr., and L. C. Feldman, "Optical properties of subwavelength hole arrays in vanadium dioxide thin films," Physical Review $B$, vol. 73, no. 20, 4 pages, 2006, Article ID 201401.

[10] H. F. Ghaemi, T. Thio, D. E. Grupp, T. W. Ebbesen, and H. J. Lezec, "Surface plasmons enhance optical transmission through subwavelength holes," Physical Review B, vol. 58, no. 11, pp. 6779-6782, 1998.

[11] T. Thio, H. F. Ghaemi, H. J. Lezec, P. A. Wolff, and T. W. Ebbesen, "Surface-plasmon-enhanced transmission through hole arrays in Cr films," Journal of the Optical Society of America B, vol. 16, no. 10, pp. 1743-1748, 1999.

[12] L. Martín-Moreno, F. J. García-Vidal, H. J. Lezec, et al., "Theory of extraordinary optical transmission through subwavelength hole arrays," Physical Review Letters, vol. 86, no. 6, pp. 1114-1117, 2001.

[13] S. A. Darmanyan and A. V. Zayats, "Light tunneling via resonant surface plasmon polariton states and the enhanced transmission of periodically nanostructured metal films: an analytical study," Physical Review B, vol. 67, no. 3, 7 pages, 2003, Article ID 035424.

[14] H. J. Lezec and T. Thio, "Diffracted evanescent wave model for enhanced and suppressed optical transmission through subwavelength hole arrays," Optics Express, vol. 12, no. 16, pp. 3629-3651, 2004.

[15] G. Gay, O. Alloschery, B. V. de Lesegno, C. O’Dwyer, J. Weiner, and H. J. Lezec, "The optical response of nanostructured surfaces and the composite diffracted evanescent wave model," Nature Physics, vol. 2, no. 4, pp. 262-267, 2006.

[16] G. Gay, O. Alloschery, B. V. de Lesegno, J. Weiner, and H. J. Lezec, "Surface wave generation and propagation on metallic subwavelength structures measured by far-field interferometry," Physical Review Letters, vol. 96, no. 21, 4 pages, 2006, Article ID 213901.

[17] G. Gay, O. Alloschery, J. Weiner, et al., "Surface quality and surface waves on subwavelength-structured silver films," Physical Review E, vol. 75, no. 1, 4 pages, 2007, Article ID 016612.

[18] P. Lalanne and J. P. Hugonin, "Interaction between optical nano-objects at metallo-dielectric interfaces," Nature Physics, vol. 2, no. 8, pp. 551-556, 2006.

[19] F. Kalkum, G. Gay, O. Alloschery, et al., "Surface-wave interferometry on single subwavelength slit-groove structures fabricated on gold films," Optics Express, vol. 15, no. 5, pp. 2613-2621, 2007.

[20] G. Gay, O. Alloschery, J. Weiner, et al., "The response of nanostructured surfaces in the near field," Nature Physics, vol. 2, no. 12, p. 792, 2006.
[21] F.J. García-Vidal, S. G. Rodrigo, and L. Martín-Moreno, "Foundations of the composite diffracted evanescent wave model," Nature Physics, vol. 2, no. 12, p. 790, 2006.

[22] P. Lalanne, J. P. Hugonin, M. Besbes, and P. Bienstman, "Reply: the response of nanostructured surfaces in the near field," Nature Physics, vol. 2, no. 12, pp. 792-793, 2006.

[23] J. Weiner and H. J. Lezec, "Reply: foundations of the composite diffracted evanescent wave model [2]," Nature Physics, vol. 2, no. 12, p. 791, 2006.

[24] A. Degiron and T. W. Ebbesen, "The role of localized surface plasmon modes in the enhanced transmission of periodic subwavelength apertures," Journal of Optics A, vol. 7, no. 2, pp. S90-S96, 2005.

[25] P. Lalanne, J. C. Rodier, and J. P. Hugonin, "Surface plasmons of metallic surfaces perforated by nanohole arrays," Journal of Optics A, vol. 7, no. 8, pp. 422-426, 2005.

[26] H. Raether, Surface Plasmons on Smooth and Rough Surfaces and on Gratings, vol. 111 of Springer Tracts in Modern Physics, Springer, Berlin, Germany, 1988.

[27] C. Genet, M. P. van Exter, and J. P. Woerdman, "Fanotype interpretation of red shifts and red tails in hole array transmission spectra," Optics Communications, vol. 225, no. 46, pp. 331-336, 2003.

[28] M. Sarrazin and J.-P. Vigneron, "Optical properties of tungsten thin films perforated with a bidimensional array of subwavelength holes," Physical Review E, vol. 68, no. 1, 4 pages, 2003, Article ID 016603.

[29] M. Sarrazin, J.-P. Vigneron, and J.-M. Vigoureux, "Role of Wood anomalies in optical properties of thin metallic films with a bidimensional array of subwavelength holes," Physical Review B, vol. 67, no. 8, 8 pages, 2003, Article ID 085415.

[30] A. Hessel and A. A. Oliner, "A new theory of woods anomalies on optical gratings," Applied Optics, vol. 4, no. 10, pp. 1275$1298,1965$.

[31] J. E. Stewart and W. S. Gallaway, "Diffraction anomalies in grating spectrophotometers," Applied Optics, vol. 1, no. 4, pp. 421-430, 1962.

[32] J. B. Goodenough, "Two components of crystallographic cransition in $\mathrm{VO}_{2}$," Journal of Solid State Chemistry, vol. 3, no. 4, pp. 490-500, 1971.

[33] M. Imada, A. Fujimori, and Y. Tokura, "Metal-insulator transitions," Reviews of Modern Physics, vol. 70, no. 4, pp. 1039-1263, 1998.

[34] S. Biermann, A. Poteryaev, A. I. Lichtenstein, and A. Georges, "Dynamical singlets and correlation-assisted Peierls transition in $\mathrm{VO}_{2}$," Physical Review Letters, vol. 94, no. 2, 4 pages, 2005, Article ID 026404.

[35] M. Rini, A. Cavalleri, R. W. Schoenlein, et al., "Photoinduced phase transition in $\mathrm{VO}_{2}$ nanocrystals: ultrafast control of surface-plasmon resonance," Optics Letters, vol. 30, no. 5, pp. 558-560, 2005.

[36] V. A. Klimov, I. O. Timofeeva, S. D. Khanin, E. B. Shadrin, A. V. Ilinskii, and F. Silva-Andrade, "Hysteresis loop construction for the metal-semiconductor phase transition in vanadium dioxide films," Technical Physics, vol. 47, no. 9, pp. 1134-1139, 2002.

[37] H. W. Verleur, A. S. Barker, and C. N. Berglund, "Optical properties of $\mathrm{VO}_{2}$ between 0.25 and $5 \mathrm{eV}$," Physical Review, vol. 172, no. 3, pp. 788-798, 1968.

[38] A. Degiron, H. J. Lezec, W. L. Barnes, and T. W. Ebbesen, "Effects of hole depth on enhanced light transmission through subwavelength hole arrays," Applied Physics Letters, vol. 81, no. 23, pp. 4327-4329, 2002. 
[39] K. L. van der Molen, F. B. Segerink, N. F. van Hulst, and L. Kuipers, "Influence of hole size on the extraordinary transmission through subwavelength hole arrays," Applied Physics Letters, vol. 85, no. 19, pp. 4316-4318, 2004.

[40] D. S. Kim, S. C. Hohng, V. Malyarchuk, et al., "Microscopic origin of surface-plasmon radiation in plasmonic band-gap nanostructures," Physical Review Letters, vol. 91, no. 14, 4 pages, 2003, Article ID 143901.

[41] K. Iizuka, Elements of Photonics, John Wiley \& Sons, New York, NY, USA, 2002.

[42] W. Bogaerts, P. Bienstman, D. Taillaert, R. Baets, and D. de Zutter, "Out-of-plane scattering in photonic crystal slabs," IEEE Photonics Technology Letters, vol. 13, no. 6, pp. 565-567, 2001.

[43] E. D. Palik, Handbook of Optical Constants of Solids, Academic Press, Orlando, Fla, USA, 1985.

[44] S. G. Tikhodeev, A. L. Yablonskii, E. A. Muljarov, N. A. Gippius, and T. Ishihara, "Quasiguided modes and optical properties of photonic crystal slabs," Physical Review B, vol. 66, no. 4, 17 pages, 2002, Article ID 045102.

[45] A. Cavalleri, C. Tóth, C. W. Siders, et al., "Femtosecond structural dynamics in $\mathrm{VO}_{2}$ during an ultrafast solid-solid phase transition," Physical Review Letters, vol. 87, no. 23, 4 pages, 2001, Article ID 237401.

[46] A. Cavalleri, Th. Dekorsy, H. H. W. Chong, J. C. Kieffer, and R. W. Schoenlein, "Evidence for a structurally-driven insulator-to-metal transition in $\mathrm{VO}_{2}$ : a view from the ultrafast timescale," Physical Review B, vol. 70, no. 16, 4 pages, 2004, Article ID 161102.

[47] C. Kübler, H. Ehrke, R. Huber, et al., "Ultrafast conductivity and lattice dynamics of insulator-metal phase transition in $\mathrm{VO}_{2}$ studied via multi-terahertz spectroscopy," in Proceedings of the Joint 31st International Conference on Infrared and Millimeter Waves and 14th International Conference on Terahertz Technology (IRMMW-THz '06), Shanghai, China, September 2006.

[48] M. F. Becker, A. B. Buckman, R. M. Walser, T. Lépine, P. Georges, and A. Brun, "Femtosecond laser excitation of the semiconductor-metal phase-transition in $\mathrm{VO}_{2}$," Applied Physics Letters, vol. 65, no. 12, pp. 1507-1509, 1994.

[49] M. F. Becker, A. B. Buckman, R. M. Walser, T. Lépine, P. Georges, and A. Brun, "Femtosecond laser excitation dynamics of the semiconductor-metal phase transition in $\mathrm{VO}_{2}$," Journal of Applied Physics, vol. 79, no. 5, pp. 2404-2408, 1996.

[50] C. Kübler, H. Ehrke, R. Huber, et al., "Coherent structural dynamics and electronic correlations during an ultrafast insulator-to-metal phase transition in $\mathrm{VO}_{2}$," Physical Review Letters, vol. 99, no. 11, 4 pages, 2007, Article ID 116401. 

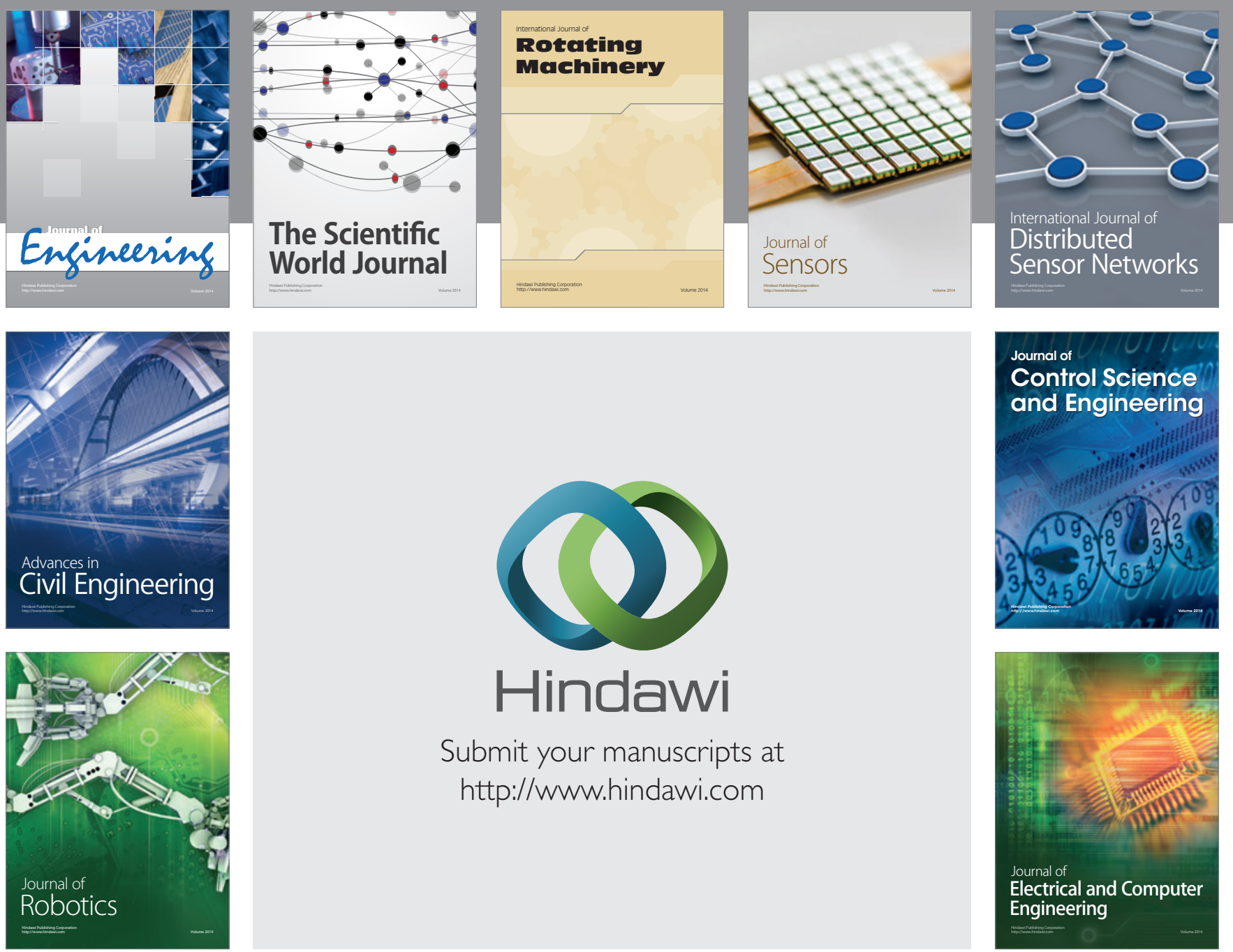

Submit your manuscripts at

http://www.hindawi.com
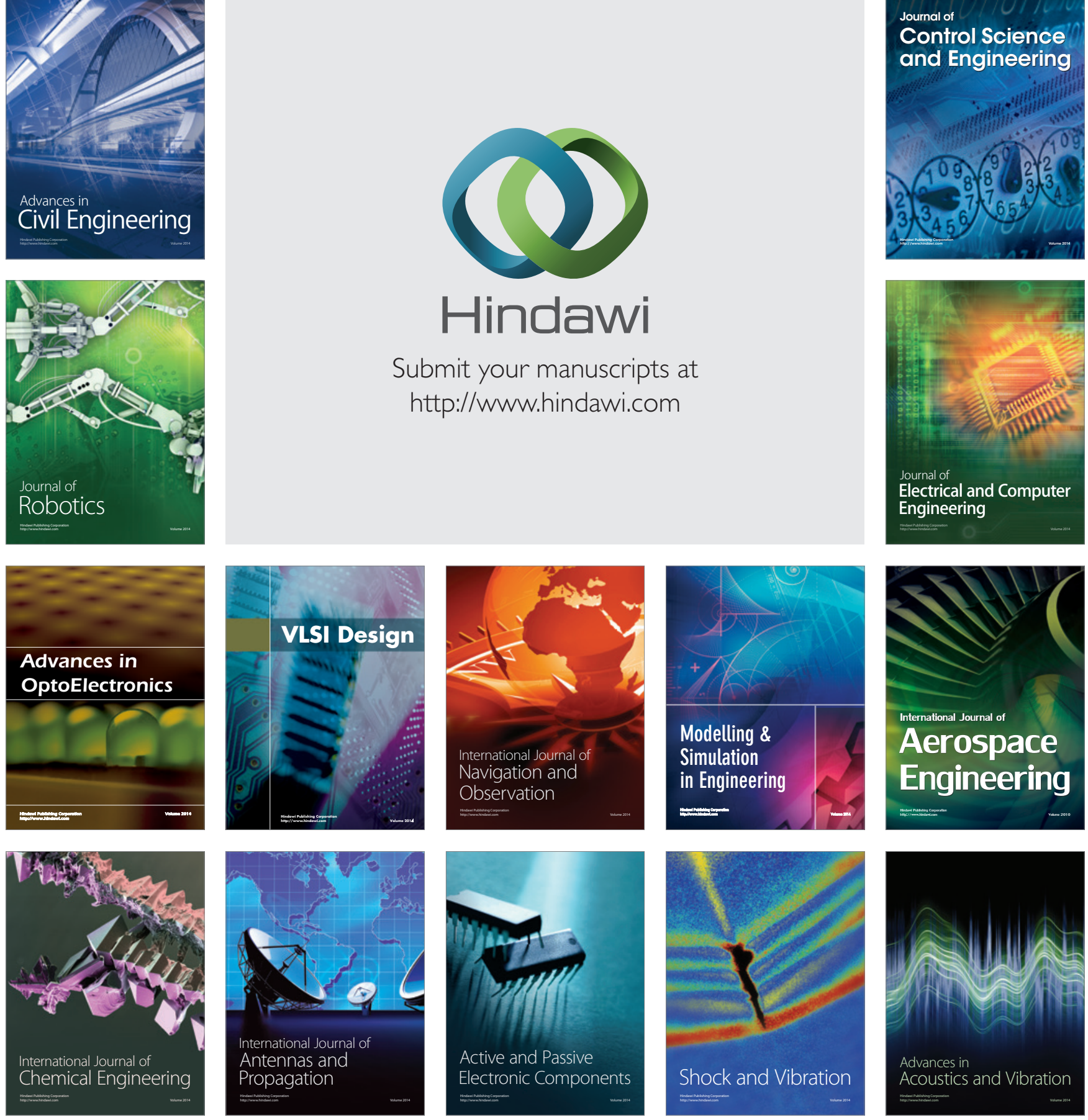